\title{
The Study on Drought Trend Evolution in Liaoning Province in recent 50 years based on SPEI
}

\author{
Yanru Liu ${ }^{1}$, Donglai Ma ${ }^{2}$, Xiaojing Liu ${ }^{1, *}$ Meixuan Huang ${ }^{1}$ \\ ${ }^{1}$ College of Tourism and Geographic Science, Jilin Normal University, Siping 136000, China \\ ${ }^{2}$ University of Tsukuba Graduate School of System and Information Engineering, Tsukuba 3050006, \\ Japan
}

\section{基于 SPEI 的辽宁省近 50 年干旱趋势演变研究 \\ 刘燕儒 ${ }^{1}$, 马东来 ${ }^{2}$, 刘晓静 ${ }^{1, *}$, 黄眉宣 ${ }^{1}$ \\ '吉林师范大学旅游与地理科学学院, 四平 136000 , 中国 \\ ${ }^{2}$ University of Tsukuba Graduate School of System and Information Engineering, Tsukuba 3050006,} Japan

\section{Abstract}

Taking Liaoning Province as the study area, Standardized Precipitation Evapotranspiration Index (SPEI) was constructed by using the daily precipitation and temperature data from 25 stations during 1965-2014. The annual precipitation and annual average SPEI were tested for by Mann-Kendall method to discuss the changes of inter-decadal drought trend in the study area. The coverage of each drought level was analyzed based on the ratios of the number of drought stations at each drought level to the total number of stations. The results show that: precipitation in Liaoning Province had dramatic changes in recent 50 years; the annual average SPEI showed a trend of first increase and then decrease; from the 1980s, the drought trend increased significantly; the coverages of all drought levels had an upward trend, and the light drought coverage had the fastest growing trend. It indicated that the frequency of drought events in the study area was increasing. This

*通讯作者: liuxj@jlnu.edu.cn study can provide decision-making basis for drought prevention and drought relief in Liaoning Province.

Keywords:SPEI;Droughttrend; Mann-Kendall; Liaoning province

摘要

以辽宁省为研究区, 利用 1965-2014 年 25 个站点的逐日降水量和气温数据, 构建年 均标准化蒸散发指数 (SPEI), 对降水和年 均 SPEI 进行 Mann-Kenda11 突变检测, 探讨 研究区年代际的干旱趋势变化, 并计算不同 等级干旱的站次比, 分析各干旱等级的覆盖 范围。结果表明: 辽宁省近 50 年降水变化 趋势剧烈; 年均 SPEI 指数呈先增长后下降 的趋势, 80 年代开始, 干旱趋势明显增强; 各干旱等级覆盖范围均呈上升趋势, 其中轻 旱覆盖范围上升趋势增长最快, 表明研究区 干旱事件发生频率越来越高。研究成果可为 辽宁省防旱抗旱提供决策依据。

关键词: SPEI; 干早趋势; Mann-Kenda11; 辽宁省

\section{1. 引言}

干旱灾害约占我国气象灾害的 50\%[1]。 
随着全球温暖化, 我国北方地区的干旱趋势 也在加剧[2]-[4]。干旱已给我国农牧业生产 和经济发展带来不可估量的损失。标准化降 水蒸散发指数 (Standardized Precipitation Evapotranspiration Index, SPEI) 和标准化降 水指数 (Standardized Precipitation Index, SPI) 是目前国际上较为典型且应用较为广泛的 干旱监测与评估指标[5] -[11]。干旱形成机理 复杂, 且受到众多因素的影响 [12]。SPI 虽 计算简便, 但却只考虑了降水, 忽略了影响 干旱的其他因素。而 SPEI 基于水分平衡原 理, 考虑了降水与蒸散发的关系, 在描述干 旱形成机理上比 SPI 更为合适。本研究利用 辽宁省 1965-2014 年逐日降水和温度数据, 计算年均 SPEI 指数, 分析辽宁省干旱年代 际特征, 揭示近 50 年辽宁省干旱趋势变化 特征, 为辽宁省干旱灾害防治提供借鉴与参 考。

\section{2. 资料与方法}

\section{1 研究区概况}

辽宁省位于我国东北地区南部(图 1), 位于东经 $118^{\circ} 50^{\prime}-125^{\circ} 47^{\prime}$, 北纬 $38^{\circ}$ $43^{\prime}-43^{\circ} 29^{\prime}$ 之间, 占地面积 14.8 万平方 公里, 毗邻渤海、黄海。辽宁省属温带大陆 性季风气候, 冬季寒冷、持续时间长, 春秋 季时间短, 受海洋和地势影响, 降水自东向
西逐渐减少, 年均气温从东北到西南逐渐增 高。近几年, 辽宁省干旱频发, 尤其在 2014 年, 辽宁省遭受了严重夏旱, 干旱面积居全 国之首。

\section{2 研究方法}

SPEI 的计算方法与 SPI 的类似, 不同的 是 SPEI 的输入因子在降水量的基础上又引 入了蒸散量。具体计算步骤如下:

计算逐月降水与蒸散的差值 Di:

$$
\mathrm{D}_{i}=P_{i}-P E I_{i}
$$

其中 $P i$ 为第 $i$ 月的降水量; PETi 为第 i 月蒸散量, 采用 Thornthwait 方法 [13]- [14] 进行计算。

建立不同时间尺度气候学意义的水分 盈/亏累积序列:

$$
\mathrm{D}_{n}^{k}=\sum_{i=0}^{k-1}\left(P_{n-i}-P E T_{n-i}\right), n \geq k \text { (2) }
$$

式中, $k$ 为时间尺度, $n$ 为计算次数;

采用 3 参数的 log-logistic 概率分布 对 Di 数据序列进行正态化, 累积函数为:

$$
\mathrm{f}(x)=\frac{\beta}{\alpha}\left(\frac{x-\gamma}{\alpha}\right)^{\beta-1}+\left[1+\left(\frac{x-\gamma}{\alpha}\right)^{\beta}\right]^{-2}
$$

公式中, $\alpha$ 为尺度参数, $\beta$ 为形状参数, $\mathrm{V}$ 为 origin 参数, 这些参数可通过 $\mathrm{L}$-矩参 数估计方法求得。于是, 给定时间尺度的累

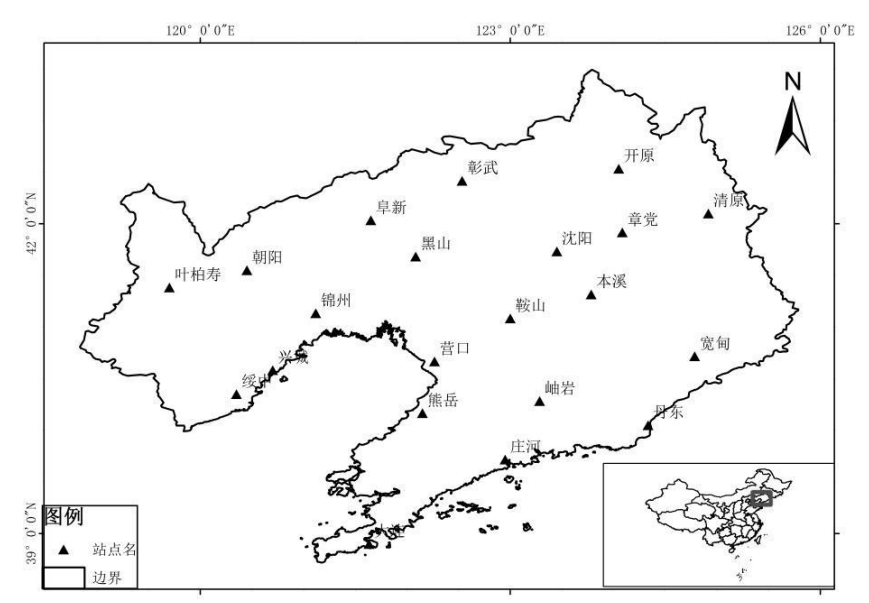

图 1 研究区位置图 
积概率可计算如下:

$$
F(x)=\left[1+\left(\frac{\alpha}{x-\gamma}\right)^{\beta}\right]^{-1}
$$

其中参数 $\alpha, \beta, x, y$ 分别采用线性矩 的方法拟合得到。

经过标准化正态分布处理求解可得:

$$
\begin{aligned}
\text { SPEI } & =\left(w-\frac{C_{0}+C_{1} w+C_{2} w^{2}}{1+d_{1} w+d_{2} w^{2}+d_{3} w^{3}}\right) （ 5 ） \\
\mathrm{w} & =\sqrt{-2 \ln (p)} \quad p \leq 0.5 \quad(6)
\end{aligned}
$$

由公式可知: $p=1-F(x)$, 当 $p>0.5$ 时, $\mathrm{w}=\sqrt{-2 \ln (\mathrm{p})}$ 中 $\mathrm{p}$ 变为 $1-\mathrm{p}$; 参数 $\mathrm{C} 0=2.515517, \mathrm{C} 1=0.802853, \mathrm{C} 2=0.010328, \mathrm{~d}$ $1=1.432788, \mathrm{~d} 2=0.182969, \mathrm{~d} 3=0.001308$ 。

由于 SPEI 与 SPI 原理相似, SPEI 指数 干旱等级的划分采用 SPI 干旱等级标准, 因 此, 依据《GB/T0481-2006 气象干早等级》 国家标准 [15], 将 SPEI 指数进行干旱分级, 如表 1 所示。

表 1 SPEI 干旱等级划分

\begin{tabular}{cc}
\hline SPEI 指数分级干早等级 & \\
\hline$-0.5<\mathrm{SPEI}$ & 正常 \\
$-1.0<\mathrm{SPEI} \leq-0.5$ & 轻旱 \\
$-1.5<\mathrm{SPEI} \leq-1.0$ & 中旱 \\
$-2.0<\mathrm{SPEI} \leq-1.5$ & 重旱 \\
$\mathrm{SPEI} \leq-2.0$ & 极端干早 \\
\hline
\end{tabular}

\section{3. 结果分析}

\section{1 辽宁省降水突变检测}

对辽宁省 50 年降水量做 Mann-Kenda11 突变检验 (图 2)。由图 2 可知, UF 和 UB 曲 线在 0.05 显著水平临界线内相交共有 9 处, 分别为 1978 年、1984 年、1988 年、1990 年、 1991 年、1993 年、1996 年、2009 年和 2014 年, 表明辽宁省降水在 1965-2014 这 50 年 内出现 9 次突变。整体来看, 辽宁省降水序 列呈现上升-下降-上升-下降的趋势, 降水 变化趋势比较剧烈。1978 年, 降水量呈大幅 下降趋势, 从1985 年开始到 1999 年, 降水 量呈先下降后增加趋势, 2000 年至 2010 年 之间, 降水序列呈现先增加后下降趋势, 2010 年至 2013 年, 降水量先下降后增加趋 势, 2013 年以后, 降水量再次下降。

\subsection{SPEI 指数年际变化及突变检测}

辽宁省 1965-2014 年平均 SPEI 指数年 际变化及其 Mann-Kenda11[16]检验(图 3)。 由图中 UF 曲线可知, 近 50 年来, 辽宁省年 均 SPEI 指数呈先增长后下降的趋势。UF 曲 线在显著水平 0.05 临界线内, 并与 UB 曲线 相交于 1980、1985 和 1988 年, 表明辽宁省 干旱趋势在这三年发生突变。1980 年开始, 年均 SPEI 呈大幅下降趋势, 干旱加剧, 1985 年发生突变后, 年均 SPEI 呈短暂上升趋势, 直至 1988 年再一次突变后, 年均 SPEI 再次 呈现下降趋势, UF 值一直小于 0 , 表明从 1988 年起, 辽宁省开始呈现干旱化趋势。从 SPEI 年际变化趋势来看, 从 80 年代开始, 干旱 趋势明显增强, 甚至从 1999 年起辽宁省连 续 5 年都发生了不同程度的干旱, 年代间平 均 SPEI 指数下降趋势显著。从 70 年代至 00 年代, 年代间平均 SPEI 指数下降速度为 0.22 , 而 90 年代至 00 年代的下降速度更高达 0.63 。 


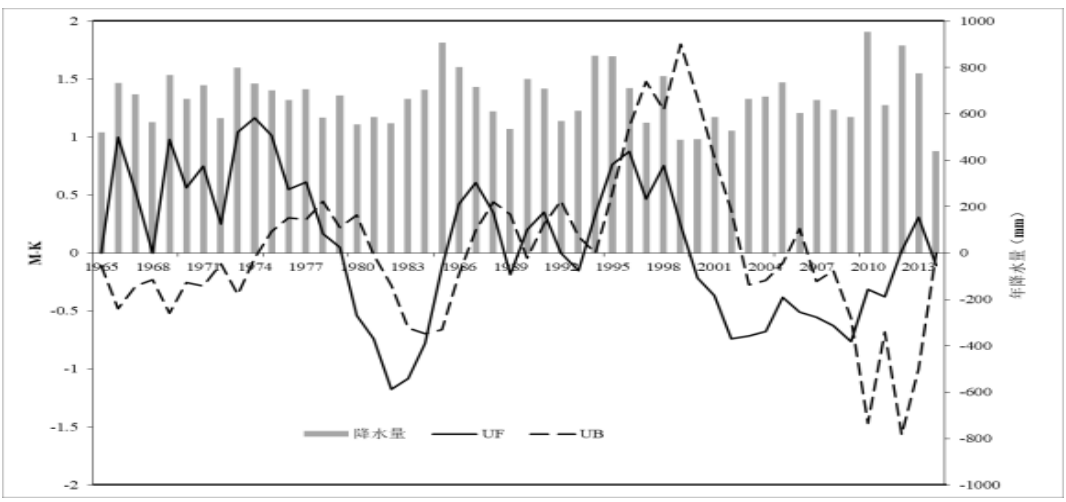

图 2 降水 $M-K$ 突变检测

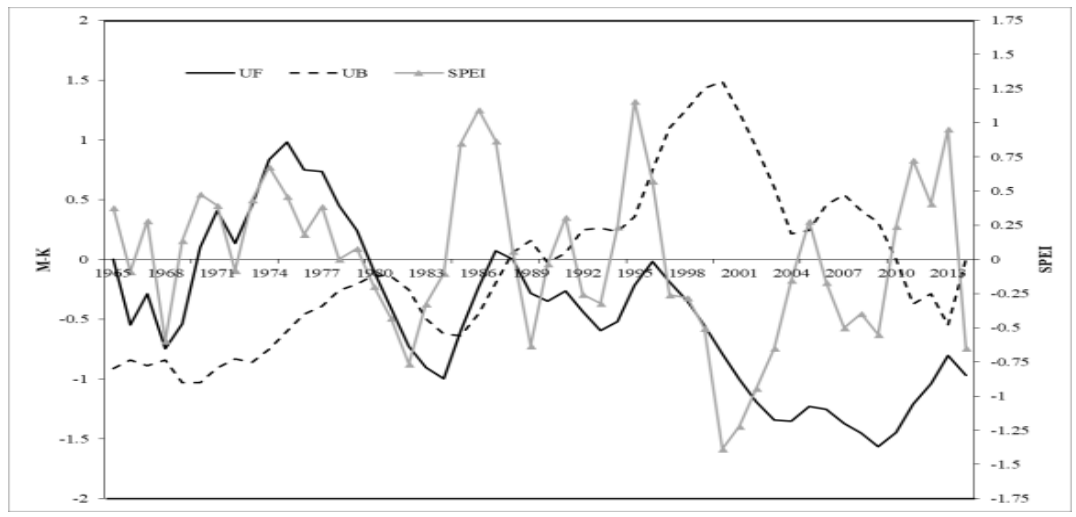

图 3 SPEI M-K 突变检测及其折线图

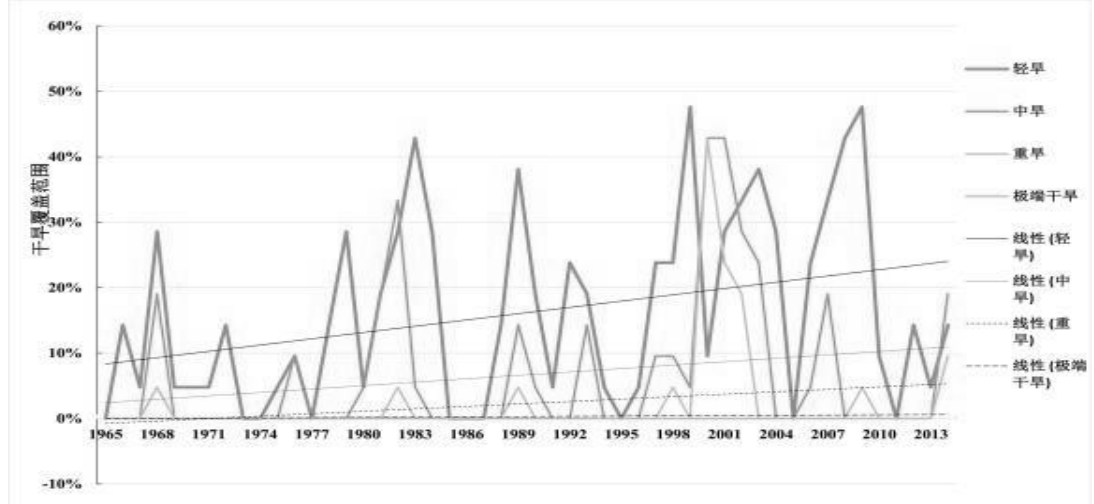

图 4 辽宁省近 50 年不同等级干旱的覆盖范围 


\section{3 不同干旱等级覆盖范围}

利用各等级干旱站点数与总站点数的 比值来代表研究区不同等级干旱的覆盖范 围 (图 4)。由图 4 可以看出, 辽宁省 1965-2014 年间各等级干旱覆盖范围均呈上 升趋势。轻旱是各站点出现最频繁的干旱等 级, 也是覆盖范围随年际变化增长最快的干 旱等级, 表明研究区干旱事件发生频率越来 越高。 70 年代、 80 年代各有 3 年未有站点 发生轻旱, 到了 90 年代、00 年代和 10 年代, 则是各有 1 年没有轻旱。重旱覆盖范围主要 分布在 1982、1989、1998、2000、2001、2002、 2009 和 2014 年, 而极端干旱覆盖范围主要 集中在 1968 年和 2014 年, 其余年份没有站 点发生极端干旱。

\section{4. 结论}

区域干旱趋势演变研究有助于地方政 府对干旱变化特征的正确认识 [17]。研究利 用近 50 年降水和气温数据构建辽宁省年均 SPEI 指数, 根据气象干旱等级国家标准, 将 SPEI 指数分为轻旱、中旱、重旱和极端干旱 四个等级, 计算不同干旱等级的覆盖范围, 并通过对降水和年均 SPEI 指数的突变检测, 分析研究区干旱趋势的年代际演变特征, 得 到以下结论:（1）整体来看, 辽宁省降水序 列呈现上升一下降一上升一下降的趋势, 降水 变化趋势比较剧烈, 从 70 年代至 00 年代, 年代间平均 SPEI 指数下降速度为 0.22 , 而 90 年代至 00 年代的下降速度更高达 0.63 ;

(2) 近 50 年来, 辽宁省年均 SPEI 指数呈 先增长后下降的趋势, 且从 1988 年起, 辽 宁省开始呈现干旱化趋势, 表明研究区干早 事件发生频率越来越高。从 SPEI 年际变化 趋势来看, 从 80 年代开始, 干旱趋势明显 增强; 从 70 年代至 00 年代, 年代间平均 SPEI 指数下降速度为 0.22 , 而 90 年代至 00 年代 的下降速度更高达 0.63。（3）1965-2014 年 间各等级干旱覆盖范围均呈上升趋势。70 年 代、 80 年代各有 3 年未有站点发生轻旱, 到 了 90 年代、 00 年代和 10 年代, 则是各有 1 年没有轻旱。轻旱是各站点出现最频繁的干 旱等级, 也是覆盖范围随年际变化增长最快 的干旱等级, 表明研究区干旱事件发生频率
越来越高。

\section{Acknowledgements}

This study was supported by National Natural Science Foundation of China (41501559, 41571491, 41501557).

\section{致谢}

本研究得到了国家自然科学基金项目 (41501559，41571491，41501557) 的资助。

\section{参考文献}

[1] 张书余.干旱气象学. 北京: 气象出版社, 2008.

[2] 马柱国,符淙斌.1951 2004 年中国北 方干旱化的基本事实.科学通报,2006. 5 0(20): 2429-2439.

[3] 章大全,张璐,杨杰,等.近 50 年中国降水 及温度变化在干旱形成中的影响.物理 学报,2010, 59(1)：655-663.

[4]谢应齐,杨子生.云南省农业自然灾害区 划.北京: 中国农业出版社,1995.

[5] Vicente-Serrano S M, S. Beguería, J. I. López-Moreno. A multiscalar drought index sensitive to global warming: the standardized precipitation evapotranspiration index. Journal of Climate, 2010, 23:1696-1718.

[6] Vicente-Serrano SM, S. Beguera, J. I. López-Moreno, M. Angulo, A. El Kenawy. A new global $0.5^{\circ}$ gridded dataset (1901-2006) of a multiscalar drought index: comparison with current drought index datasets based on the palmer drought severity index. Journal of Hydrometeor,2010, 11: 1033-1043.

[7] McKee T B, Doesken N J, Kleist J. The relationship of drought frequency and duration to time scales. Eighth Conference on Applied Climatology, 1993: 179-184.

[8] McKee $\mathrm{T}$ B, Doesken $\mathrm{N}$ J, Kleist J. Drought monitoring with multiple time scales. Proceedings of the Ninth 
Conference on Applied Climatology. 1995: 233-236.

[9] Palmer W C. Meteorological Drought: Research Paper No. 45. U. S. Depa rtment of Commerce Weather Bureau: Washington, DC, USA, 1965.

[10] 石崇, 刘晓东.1949-2006 年东半球陆地 干旱化特征一基于 SPEI 数据的分析. 中国沙漠,2012,32 (6): 1691-1701.

[11] 张继权,李宁.主要气象灾害风险评价与 管理的数量化方法及其应用. 北京: 北京 师范大学出版社,2007.

[12] 刘晓静,温馨. 基于 SPI 指数的近 50 年辽 宁省干旱时空演变规律分析. 吉林师范 大学学报(自然科学版),2016,37(02):146 -151 .

[13] 轩俊伟,郑江华,刘志辉. 基于 SPEI 的新 疆干旱时空变化特征.干旱区研究,2016, 33(02):338-344.
[14] 苏宏新,李广起. 基于 SPEI 的北京低频干 旱与气候指数关系.生态学报,2012,32(1 7):5467-5475.

[15] 李龙,徐玲玲,方军.三种气象干旱等级评 价方法的比较与改进.中国农业气象, 20 09,30(S2):310-312.

[16] 于延胜,陈兴伟.基于 Mann-Kendall 法的 水文序列趋势成分比重研究.自然资源 学报,2011,26(09):1585-1591.

[17] Chen X F ,Sun H H, Yuan Z, et al. Flood disaster risk assessment and spatial distribution characteristics along the Yangtze River in Anhui Province. Journal of Risk Analysis and Crisis Response 2014, 4(4): 238-242. 